\title{
Inelastic neutron scattering applied to the investigation of collective excitations in topologically disordered matter
}

\author{
J.-B.Suck \\ Institute of Physics, University of Technology Chemnitz, D-09107 Chemnitz, Germany
}

Received November 22, 2007, in final form December 17, 2007

Inelastic neutron scattering techniques are introduced here as one of the most important experimental techniques in the investigation of collective excitations in fluids (liquids and compressed gases) and amorphous solids. The correlation functions involved, the spectra of which are determined in inelastic neutron scattering experiments, the dispersion relations of the collective excitations and how they are obtained from the measured spectra and finally two of the most often used instrumental techniques are briefly discussed.

Key words: inelastic neutron scattering, correlation functions, dispersion relations, collective excitations, topologically disordered matter

PACS: $61.12 . E x, 63.20 . D j, 63.50 .+x$

\section{Introduction}

In a similar manner as inelastic neutron scattering (INS) has played a decisive role in the experimental investigation of phonon dispersions in crystal lattices in the second half of the last century, it also has played the main role in the experimental studies of the collective excitations in topologically disordered matter such as fluids and amorphous solids in the same period. Light scattering has for a long time been used in order to investigate the atomic dynamics in disordered matter next to $\vec{q}=0$, where $\vec{q}$ is the wave-vector of the excitation. However, the application of highly resolving Brillouin scattering spectrometers [1], which makes it possible to determine the dispersion and the damping of the modes with high accuracy [2], has also shown quite a durable period of their development. After the development of inelastic X-ray scattering (IXS) spectrometers by the end of the last century, this technique was very successfully applied to the investigation of collective excitations in topologically disordered matter as well [3,4]. Inelastic light and X-ray scattering techniques are described in detail in separate articles in this volume. Here the focus is on inelastic neutron scattering techniques in its application to the investigation of the collective excitations in disordered matter. Further articles in this volume will concentrate on the results obtained using this method. A more extensive discussion including results is given elsewhere [5].

\section{Neutron scattering}

What advantage does scattering of cold, thermal and epithermal neutrons have in this context? The neutron is neutral and therefore its interaction with condensed matter is weaker in a scattering experiment than that of charged particles such as electrons. This permits to use solid sample containers and even solid pressure cells, if the material is appropriately chosen. On the other hand, the reduced interaction also imposes the use of larger samples, often several $\mathrm{g}$ of a substance, and the small absorption frequently leads to a non-negligible amount of multiply scattered neutrons.

Neutrons are scattered either from the nucleus of an atom (nuclear scattering) or from the electron shell of the atom (magnetic scattering). In spite of the fact that the magnetic scattering of thermal neutrons is of primary importance in the studies of the magnetic structure and the spin 
dynamics, there have been relatively few investigations of collective magnetic excitations (spin waves) in disordered matter. Therefore, we shall concentrate on nuclear scattering.

In this case one does not have to deal with form factors as one does in magnetic scattering and in case of all the other probes, which are scattered from the electron shell of the atom. Thus, the interaction of neutrons with a wavelength of the order of $0.1 \mathrm{~nm}$ with the sample nuclei (with a diameter of some $\mathrm{fm}$ ) reduces to the scattering constant, b (scattering cross-section $\sigma^{\text {sc }}=4 \pi b^{2}$ ), which varies in a non-systematic way from element to element and even from isotope to isotope of the same element. In addition to this, the scattering is also spin dependent. While nuclear scattering is coherent, if all scatterers are identical, isotopic mixtures and spin dependent scattering can lead to incoherent scattering. If the scattering lengths, $b_{i}, b_{j}$, of the atoms at the positions $r_{i}, r_{j}$ in the sample are uncorrelated, which is most often the case, one can separate the information on the structure and dynamics of the sample from its interaction with the probe [6]. Due to this simple separation and the very good knowledge of the scattering lengths it is also possible to normalize the data very reliably and to produce results on absolute scale. This is one of the greatest advantages in the present context, as the experimental results normally turn out to be rather smooth functions and absolute values are an advantage as a further important parameter in comparison with theories.

The non-systematic variation of the scattering length from element to element and from isotope to isotope also allows us to measure light elements aside from heavy elements and to mark elements by using different isotopes (isotopic substitution) of the same element in different measurements. This possibility is used to determine the partial dynamic structure factors of multi-component systems.

A further advantage of NIS comes from the fact that the energy of cold, thermal and epithermal neutrons, depending onto which neutron moderator the beam tube used is directed, just spans the range of the energy of the excitations in fluids and amorphous solids. Thus, the incident energy, $E_{0}$, of the neutrons can be optimized to the excitation under investigation and the energy transfer, $\hbar \omega$ is then of the same order as $E_{0}$ and can therefore be highly resolved. Likewise the de Broglie wavelength is of the same order as are the atomic distances in condensed matter $(0.3 \mathrm{~nm})$. Thus, the wavelength dependence of the atomic dynamics can be measured with good resolution as well.

Due to its large mass, even small changes of the neutron velocity, $\vec{v}$, can be connected with large momentum transfers, $\hbar \vec{Q}$. Therefore, INS is most favorably used for investigating the

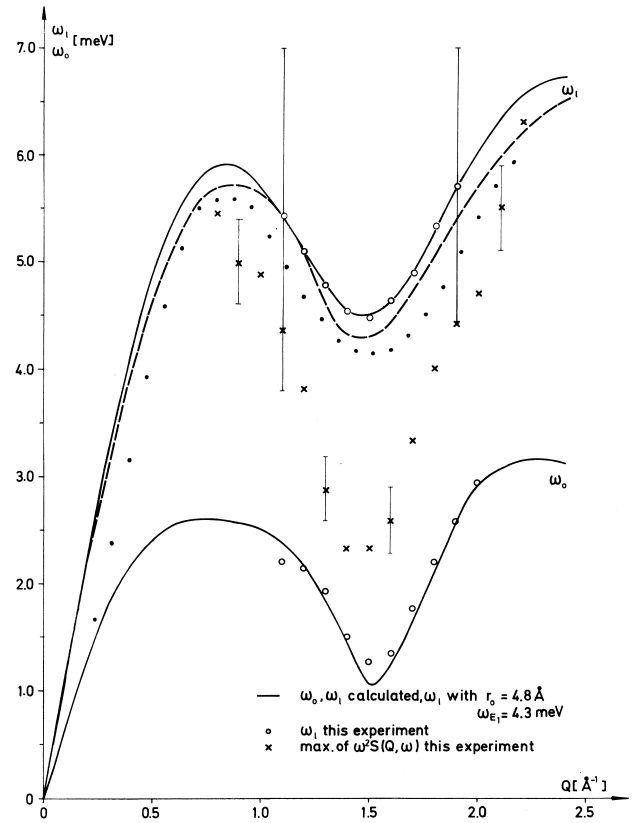

Figure 1. The adiabatic dispersions for liquid $\mathrm{Rb}$ from experiment and model calculations (lower dispersion). The high frequency dispersion from experiment, model calculations [9] and computer simulations [10] (upper dispersion). The crosses between these two dispersions were obtained from the maxima of $J_{1}(Q, \omega)$. atomic structure and dynamics far out into reciprocal space within the higher order Brillouin zones (BZ) of the reciprocal lattice of single crystals, i.e., using large wave-vector transfers $\vec{Q}=2 \pi \vec{\tau} \pm \vec{q}$. Here $2 \pi \tau$ is the vector to the reciprocal lattice point, from which the dispersion is measured. Thus, the large momentum transfer connected even with a small energy transfer gives plenty of freedom in order to optimally chose the BZ, in which one wants to measure the phonon dispersion in single crystals, - normally a further advantage of inelastic neutron scattering.

Indications for the existence of 'smeared' out reciprocal lattice 'points' at the maxima of the continuous static structure factor, $S(Q)$ and low order analogues of BZ, called pseudo Brillouin 


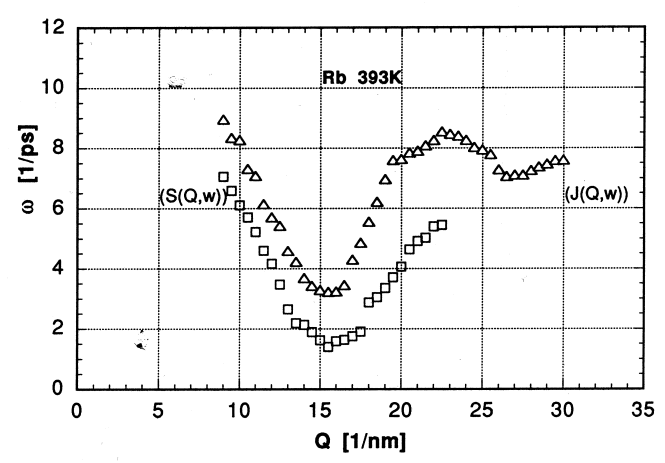

Figure 2. Dispersion of collective excitations in liquid $\mathrm{Rb}$ measured near its triple point: due to the shift of the maxima of the excitations to higher energies on multiplication with $\omega^{2}$ the dispersion obtained from $J_{l}(Q, \omega)$ has the same shape as the dispersion obtained (by a fit of a model) from $S(Q, \omega)$ but is shifted to higher energies.

zones (PBZ), exist also in disordered systems as it is suggested experimentally by the shape of the observed dispersion relations (see e.g. figures 1,2). However, these are of little help (compared to the case of single crystals mentioned above) as the dispersion of collective excitations in disordered systems is measured as a function of the wave-vector transfer, $\vec{Q}$, and not of the wave-vector of the excitation, $\vec{q}$. Only at smallest $\mathrm{Q}$ this can be identified with q. In addition, collective excitations in topologically disordered condensed matter are best investigated at small $\vec{Q}$, i.e., within the first PBZ by Neutron Brillouin Scattering (NBS) (see chapter on NBS in this volume), because there, as all computer simulations and experiments show, the collective excitations are best defined and therefore most easily separated from the foot of the elastic (amorphous samples) or quasielastic (fluids) line. For this reason, the usual advantage of a large neutron mass becomes to some extent a disadvantage in the investigation of collective excitations in disordered matter with severe consequences for the experiments (see section 5).

As in other scattering experiments, in INS one measures the intensity of the scattered neutrons, $I\left(\overrightarrow{k_{0}} \vec{k}\right)$, as a function of the wave vector of the incident, $\overrightarrow{k_{0}}$, and of the scattered neutron, $\vec{k}$, and of the incident neutron intensity on the sample, $I_{0}$. From these quantities the double differential scattering cross-section, $\mathrm{d}^{2} \sigma / \mathrm{d} \Omega \mathrm{d} E$ is calculated, which still contains the information on the sample properties and of the neutron-sample interaction. Here $\sigma, \Omega, \mathrm{d} E$ are the scattering cross-section, the solid angle under which the detector surface appears at the centre of the sample and $\mathrm{d} E$ is the energy bin, where the scattered neutron is recorded. To obtain absolute intensities, numerous corrections have to be performed, partly from measurements done under conditions identical to the sample measurement (like Cadmium-, empty can- and Vanadium calibration measurements), partly from analytical calculations (like energy and scattering angle dependent absorption corrections and the energy dependence of the detector efficiency) and partly from e.g. a Monte Carlo computer simulation (for multiple scattering corrections).

The contribution $I_{\mathrm{c}}$ from the neutrons scattered from the empty sample container to the scattered intensity $I_{\mathrm{sc}}$ in the sample measurement is obtained in the empty can measurement. Assuming absorption of all neutrons falling on the Cd-sample with the same geometry as the sample, in the Cd-measurement, the background $I_{\mathrm{Cd}}$ passing aside the sample and therefore not attenuated by the sample and its container is measured. Having normalized all the measurements to the same incident intensity (the same monitor count rate), this intensity is directly subtracted from $I_{\mathrm{sc}}$ and $I_{\mathrm{c}}$, while in the subtraction of the empty can intensity the absorption of neutrons in the sample has to be taken into account by the absorption factor $A_{\mathrm{ec}}(\theta, E)$. Here $\theta$ is the scattering angle (angle between $\overrightarrow{k_{0}}$ and $\vec{k}$ ).

After calculating the sample absorption, $A_{\mathrm{s}}(\theta, E)$, that of the empty can, $A_{c}(\theta, E)$, and the detector efficiency, $\epsilon(\theta, E)$, the intensity $M S(\theta, E)$ of the multiply scattered neutrons has still to be calculated (analytically or (better) in a computer simulation [7] of the experiment), because 
only for neutrons scattered once in the sample, $\overrightarrow{k_{0}}$ and $\vec{k}$ can be determined and hereby the energy - and momentum of the excitation in the sample

$$
\begin{aligned}
\hbar \omega & =E_{0}-E=\hbar^{2} /(2 m)\left(k_{0}{ }^{2}-k^{2}\right), \\
\hbar \vec{Q} & =\hbar\left(\vec{k}_{0}-\vec{k}\right), \\
Q & =\sqrt{{k_{0}}^{2}+k^{2}-2 k_{0} k \cos \theta} .
\end{aligned}
$$

As disordered systems are normally isotropic, in what follows the modulus of $\vec{Q}$ will be used in place of the corresponding vector for the wave-vector transfer. After the rather involved multiple scattering correction, which one tries to keep small by using sample geometries, which do not scatter more than 10 to $15 \%$ of the neutron beam incident on the sample, the fully corrected intensity from the sample, $I_{\text {corr }}$ can be calculated

$$
\begin{aligned}
\text { Subtraction of Cd }- \text { intensity : } & I_{\mathrm{sc}}-I_{\mathrm{Cd}} \\
& I_{\mathrm{c}}-I_{\mathrm{Cd}} \\
\text { Subtraction of empty can scattering : } & I_{\mathrm{s}}=I_{\mathrm{sc}}-A_{\mathrm{ec}}(\theta, E) I_{\mathrm{c}} \\
& I_{\mathrm{corr}}=\frac{I_{\mathrm{s}}-M S(\theta, E)}{N_{\mathrm{s}} \epsilon(\theta, E) A_{\mathrm{s}}(\theta, E) A_{c}(\theta, E)},
\end{aligned}
$$

where $N_{\mathrm{s}}$ is the number of scattering units calculated from the number density, n, of the sample and its volume.

The Vanadium calibration run and the V-holder measurements are done under identical conditions as the sample measurements (the same sample geometry, the same experimental conditions) and the same type of corrections are applied to the measured intensity as in the case of the sample experiment. As V scatters neutrons almost exclusively incoherently (spin incoherence), $I_{\text {corr } \mathrm{V}}$ is used for the calibration of the relative efficiencies, if several different detectors are used in the spectrometer, and for the normalization of the sample intensity to the known cross-section of $\mathrm{V}$ in the calculation of the absolute values of the double differential scattering cross-section

$$
\frac{\mathrm{d}^{2} \sigma}{\mathrm{d} \Omega \mathrm{d} E}=\frac{I_{\text {corr }}}{N_{\mathrm{su}} \Delta \Omega \Delta E I_{\text {corr } \mathrm{V}}} .
$$

Hence, the desired information on the dynamics of the sample, the dynamic structure factor $S(Q, \omega)$ can be separated from the neutron sample interaction under the conditions mentioned above [6]:

$$
S_{\exp }(Q, \omega)=\frac{4 \pi}{\sigma^{\mathrm{sc}}} \frac{k_{0}}{k} \frac{\mathrm{d}^{2} \sigma}{\mathrm{d} \Omega \mathrm{d} E},
$$

where $\sigma^{\text {sc }}$ is the total scattering cross-section of the defined scattering unit. What really $S_{\exp }(Q, \omega)$ in equation 6 represents, depends on the correlations covered in the experiment. If only heterocorrelations contribute to $S_{\exp }(Q, \omega)$, i.e., purely coherent scattering contributes to $\mathrm{d}^{2} \sigma / \mathrm{d} \Omega \mathrm{d} E$, then $\sigma^{\mathrm{sc}}=\sigma^{\mathrm{coh}}$ and $S_{\exp }(Q, \omega)=S(Q, \omega)$, the dynamic structure factor. That is the information one needs to determine the dispersion of collective excitations. Therefore one tries, if possible, to maximize the coherent part of the scattered intensity by choosing appropriate elements. If only auto-correlations contribute, i.e., purely incoherent scattering contributes to $\mathrm{d}^{2} \sigma / \mathrm{d} \Omega \mathrm{d} E$, then $\sigma^{\mathrm{sc}}=$ $\sigma^{\text {incoh }}$ and $S_{\exp }(Q, \omega)=S_{\mathrm{s}}(Q, \omega)$, the self-part of the dynamic structure factor. In most cases both correlations will contribute and it then depends on the amount of incoherent scattering, whether one has to calculate the incoherent contribution from an appropriate model and subtract this (correctly normalized) from $S_{\exp }(Q, \omega)$ to obtain the desired contribution from hetero-correlations only (see below) or one has to interpret the coherent scattering 'on top' of the incoherent 'background'.

In addition, what kind of dynamic structure factor one has determined depends on the number of different elements in the sample. If this contains one element only, the dynamic structure factor is determined (taking a weighted mean value over the scattering length of the isotopes of the element). If several different elements are present, of which each couples with a different strength 
(scattering length) to the incident neutron, the total dynamic structure factor is determined, which is the weighted sum over the partial dynamic structure factors, $S_{i, j}(Q, \omega)$

$$
\sigma S(Q, \omega)=4 \pi \sum \sum b_{i} b_{j} \sqrt{c_{i} c_{j}} S_{i, j}(Q, \omega)+\sum \sigma_{i}^{\text {inc }} c_{i} S_{i}^{s}(Q, \omega), \quad(i, j=1 \ldots n),
$$

where $c_{i}, \sigma_{i}^{\text {inc }}$ and $S_{i}^{\mathrm{s}}(Q, \omega)$ are the relative concentration, the incoherent scattering cross-section and the self-part of the dynamic structure factor (auto-correlations) of the element i. One has to realize that the dispersions obtained from the total dynamic structure factor (as was done in nearly all cases up till now) and those determined the same way from the partial dynamic structure factors (after separating them out via isotopic substitution) will not be the same in most cases.

$S(Q, \omega)$, a real function, is not symmetric on the energy scale, since the excitation of a mode is always more likely than its absorption. This detailed balance condition $S(Q, \omega)=\mathrm{e}^{\beta} S(-Q,-\omega)$, with $\beta=\hbar \omega / k_{\mathrm{B}} T$ and $k_{\mathrm{B}}$ the Boltzmann constant, is often used to symmetrize (artificially) the dynamic structure factor

$$
\tilde{S}(Q, \omega)=\mathrm{e}^{\frac{-\beta}{2}} S(Q, \omega) .
$$

This symmetrized representation is sometimes very helpful if one wants to obtain the dispersion of the collective excitations from the neutron energy gain and loss side of $S(Q, \omega)$ under most similar conditions, (see figure 3). However, one has to take care to include the symmetrization factor explicitly in all further calculations when using $\tilde{S}(Q, \omega)$.

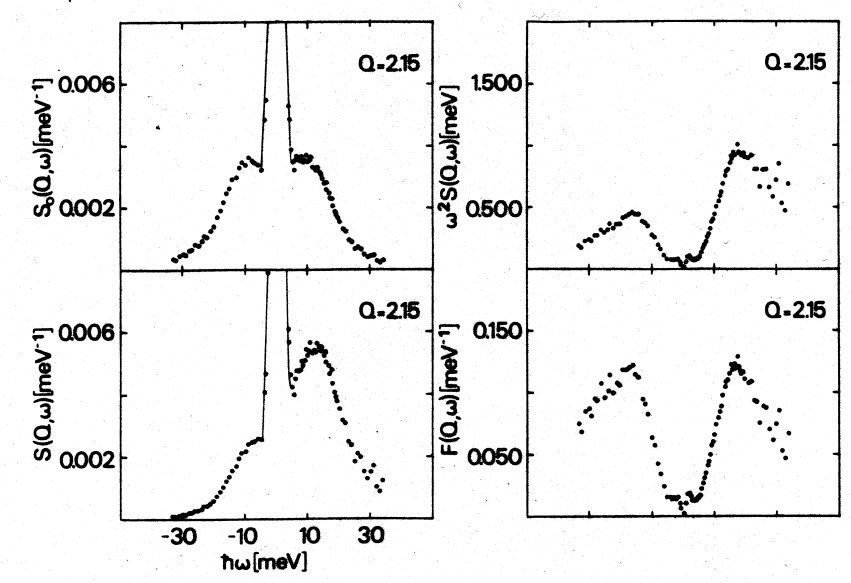

Figure 3. left from below: $S(Q, \omega), \tilde{S}(Q, \omega)$, right from above: $J_{1}(Q, \omega)$ and $F(Q, \omega)$ for the same cut at $Q=21.5 \mathrm{~nm}^{-1}$ through the spectra of the metallic glass $M g_{70} Z n_{30} . F(Q, \omega)$ is strongly approximated as it still contains contributions from incoherent scattering and multi-excitations.

\section{Correlation functions}

$S(Q, \omega)$ is the spectrum of the van Hove correlation function $G(r, t)[6,8]$, the time dependent correlation function of a pair of local densities

$$
\begin{aligned}
& n(r, t)=\frac{1}{\sqrt{N}} \sum_{1}^{N} \delta\left(\vec{r}-\vec{R}_{\mathrm{l}}(t)\right), \\
& n(Q, t)=\frac{1}{\sqrt{N}} \sum_{1}^{N} \mathrm{e}^{\mathrm{i}\left(\vec{Q} \cdot \vec{R}_{1}(t)\right)},
\end{aligned}
$$

where the second line is the local density after Fourier transformation.

$$
G(r, t)=\frac{1}{n}\left\langle\sum_{l, l^{\prime}} \delta\left(\vec{r}^{\prime}-\vec{R}_{l^{\prime}}(0)\right) \delta\left(\vec{r}-\vec{R}_{\mathrm{l}}(t)\right)\right\rangle,
$$


where $\mathrm{n}$ is the number density $(n=N / V)$ and $r=\left|\vec{r}-\vec{r}^{\prime}\right|$. Thus, in the van Hove correlation function, two particles or local densities at $R_{l}$ and $R_{l^{\prime}}$ in a distance $\mathrm{r}$ are correlated at two different times, herein 0 and $t$. If the correlated entities are different particles, then $G(r, t)=G_{d}(r, t),(\mathrm{d}=$ distinct correlation) describing the collective dynamics, which is reflected in the coherent neutron scattering. If the same particle is correlated at two different times, the single particle dynamics is reflected in $G_{\mathrm{s}}(r, t)$ (s = self-correlation) and the incoherent scattering.

$S(Q, \omega)$ and $G(r, t)$ are related to each other by double Fourier transforms

$$
\begin{aligned}
S(Q, \omega) & =\int_{-\infty}^{\infty} \mathrm{d} t \mathrm{e}^{-\mathrm{i} \omega t} \int \mathrm{d} r \mathrm{e}^{\mathrm{i}(\vec{Q} \cdot \vec{r})} G(r, t), \\
S(Q, \omega) & =\int \mathrm{d} t \mathrm{e}^{-\mathrm{i} \omega t} F(Q, t), \\
F(Q, t) & =\int \mathrm{d}^{3} r \mathrm{e}^{\mathrm{i}(\vec{Q} \cdot \vec{r})} G(r, t),
\end{aligned}
$$

where the Fourier transformation of $G(r, t)$ into Q-space (as is the Fourier transform of $S(Q, \omega)$ from energy into time space) is called the intermediate scattering function, $F(Q, t)$. In most cases, the modelling of the dynamics does not start from $G(r, t)$, the correlation function calculated in general from the results of molecular dynamics calculations (MD), nor from the experimentally determined spectrum of it, $S(Q, \omega)$, but from $F(Q, t)$, which is the correlation function of the Fourier transformed dynamical variables (see equation 10) and can be obtained from either of the functions by one Fourier transformation

$$
F(Q, t)=\left\langle n^{*}(\vec{Q}, 0) n(\vec{Q}, t)\right\rangle\left(-n(2 \pi)^{3} \delta(\vec{Q})\right) .
$$

In this equation, the second term, the forward scattering term, is normally neglected. Thus $F(Q, t)$ is a central function at which the results from theory, computer simulations and experiments, done in a sufficiently large energy range and with good statistical accuracy, best meet. In the classical approximation it describes the time decay of the correlations represented by the static structure factor of the system at $\mathrm{t}=0$, i.e., $F(Q, 0)=S(Q)$.

As the scattering units are moving, in place of the local densities one can also use the time dependent local currents $\vec{j}(\vec{r}, t)$ as dynamical variable

$$
\begin{aligned}
\vec{j}(\vec{r}, t) & =\frac{1}{\sqrt{N}} \sum_{1}^{N} \vec{v}_{1}(t) \delta\left(\vec{r}-\vec{R}_{1}(t)\right), \\
j_{\alpha}(Q, t) & =\frac{1}{\sqrt{N}} \sum_{l=1}^{N} v_{l, \alpha} \mathrm{e}^{\mathrm{i}\left(\vec{Q} \cdot \vec{R}_{1}(t)\right)}, \quad(\alpha=x, y, z) .
\end{aligned}
$$

Hence, the current correlation function, $J(r, t)$, the corresponding intermediate scattering function, $J(Q, t)$, and its spectrum, $J(Q, \omega)$, are deduced just the same way as it was done for the density as dynamical variable, except that now velocity vectors are dealt with, which have different directions in space, and which have to be correlated as well. Consequently, the corresponding correlation function now splits up additionally into longitudinal and transverse correlations.

$$
J_{\alpha \beta}(r, t)=V\left\langle j_{\alpha}\left(\vec{r}^{\prime}, 0\right) j_{\beta}(\vec{r}, t)\right\rangle, \quad(\alpha, \beta=x, y, z, \text { and } V \text { the volume }) .
$$

As in the case of the dynamic structure factor, the spectrum of the current correlation function is obtained by Fourier transformation of the corresponding intermediate scattering function $J(Q, t)$

$$
\begin{aligned}
J_{\alpha \beta}(Q, t) & =\left\langle j_{\alpha}^{*}(\vec{Q}, 0) j_{\beta}(\vec{Q}, t)\right\rangle \\
J_{\alpha \beta}(Q, t) & =\frac{Q_{\alpha} Q_{\beta}}{Q^{2}} J_{1}(Q, t)+\left(\delta_{\alpha \beta}-\frac{Q_{\alpha} Q_{\beta}}{Q^{2}}\right) J_{t}(Q, t),
\end{aligned}
$$


where $\delta_{\alpha \beta}$ is the Kronecker $\delta$. Neutrons (and X-rays) couple directly only to longitudinal motions (parallel to $\vec{Q}$ ), to transverse motions only via (for topologically disordered matter: diffuse) Umklapp scattering. Therefore for INS (and IXS) experimentally accessible is only the longitudinal part of the spectrum, $J_{l}(Q, \omega)$, of the current correlation function

$$
J_{l}(Q, \omega)=\int_{-\infty}^{\infty} \mathrm{d} t \mathrm{e}^{-\mathrm{i} \omega t} J_{l}(Q, t),
$$

while in computer simulations, the transverse part of it can be determined as well. As only one scattered intensity is recorded, from which $S(Q, \omega)$ and $J_{l}(Q, \omega)$ are calculated, the spectrum of the current correlation function has to be connected to the spectrum of the density correlation function $[6,8]$

$$
J_{l}(Q, \omega)=\frac{\omega^{2}}{Q^{2}} S(Q, \omega)
$$

In spite of this fact, $J_{1}(Q, \omega)$ plays a central role in the experimental determination of dispersion relations in disordered matter. Due to the multiplication of $S(Q, \omega)$ with $\omega^{2}$ and due to the fact that $S(Q, \omega)$ falls to zero with $\omega$ faster than any power of $\omega, J_{1}(Q, \omega)$ has always one or more maxima, which emphasize the weak and broad maxima in the inelastic part of $S(Q, \omega)$. However, one has to interpret these maxima with some caution, as the function is forced to show at least one maximum on the neutron energy gain and loss side of the spectrum, - even for a Lorentzian.

\section{Dispersion relations}

How can the dispersion of collective excitations in disordered systems be obtained from the measured spectra of the density or the current correlation functions? In principle, most methods mentioned herein below can be applied to fluids and amorphous solids. However, in practice some of them have been applied with preference to fluids, - for which presently a larger amount of different results have been obtained - the others with preference to amorphous solids.

In models, describing the collective dynamics of disordered systems such as fluids, dispersion relations defined by the ratio of subsequent lowest even frequency moments of the dynamic structure factor, $\left\langle\omega^{n}\right\rangle$, are most frequently used.

$$
\left\langle\omega^{n}\right\rangle=\int_{-\infty}^{\infty} \omega^{n} S(Q, \omega) \mathrm{d} \omega .
$$

The adiabatic ( $=$ isothermal for $\gamma=C_{p} / C_{V} \approx 1$ ) dispersion $\omega_{0}(Q)$ is the ratio of the second to the zeroth frequency moment, $k_{\mathrm{B}} T Q^{2} / M$ and $S(Q)$, where $\mathrm{M}$ is the mass of the scattering unit. The high frequency dispersion, $\omega_{\mathrm{l}}(Q)$, is the ratio of the fourth to the second moment [6].

$$
\begin{aligned}
\omega_{0} & =\frac{k_{\mathrm{B}} T Q^{2}}{M S(Q)}, \\
\omega_{l}^{2} & =3 \frac{k_{\mathrm{B}} T Q^{2}}{M}+\Omega^{2}(0)-\Omega^{2}(Q), \\
\Omega^{2}(Q) & =\frac{n}{M} \int \mathrm{d}^{3} r g(r) \cos (Q z) \frac{\mathrm{d}^{2} \phi(r)}{\mathrm{d} z^{2}},
\end{aligned}
$$

where $\phi$ is the inter-atomic potential. While the interaction potential enters the adiabatic dispersion only indirectly via $S(Q)$, the high frequency dispersion also contains, besides the free particle dispersion (first term in the high Q limit) in its second and third terms, a potential part (similar to the dynamical matrix for harmonic system, but for the topologically disordered system weighted with the particle distribution according to the radial distribution function $\mathrm{g}(\mathrm{r})$ ). Here the effect of the potential on the dynamics comes in explicitly. The two parts of the high frequency dispersion react very differently on temperature and pressure changes. While the free particle dispersion reacts 
most strongly on the changes in temperature, the changes in pressure (and therefore in density) have much greater effect on the potential part of the dispersion [12].

One can determine these dispersions either from the ratio of experimentally determined frequency moments, as it was first done for liquid $\mathrm{Rb}$ (see figure 1) and later for fluid $\mathrm{Ar}$ [11] or from a fit of a model, where these dispersions are treated as parameters [12], or from models for the frequency moments. The inclusion of these dispersions in the (visco-elastic) models is very important, because they guarantee that the model fulfils the condition of the existence of all frequency moments up to the fourth.

A more straightforward approach is to fit the models for the collective atomic dynamics to the dynamic structure factor and determine the position (energy) and the width (damping) of the maxima or shoulders corresponding to the excitations. If the data were taken in an $Q-\omega$ range corresponding to the hydrodynamic limit, hydrodynamic equations (essentially three Lorentzians with two S-shaped additions) or kinetic or linear response theory, taken in the hydrodynamic limit (all parameters are constants, for an explicit comparison see [13]), can be used.

If the data were taken outside the hydrodynamic limit, one can fit generalized hydrodynamic models (the same equations but parameters become functions of $\mathrm{Q}$ (and $\omega$ )) e.g. kinetic or linear response theory or mean-field (visco-elastic) models to the dynamic structure factor. Models for binary systems do exist [14], but they have got quite a few (not necessarily independent) parameters.

Starting from the local current as dynamical variable, one can fit convex functions to the maxima of $J_{1}(Q, \omega)$ and determine the dispersion from these peak positions. Only if the excitations can be represented by $\delta$-functions, as would be the case for ideal phonons with an infinite lifetime, the dispersion obtained this way will be identical to the dispersion determined by fitting the inelastic maxima in $S(Q, \omega)$. As shown in figure 2, for excitations in topologically disordered systems the dispersion relations have approximately the same shape (as a function of $\mathrm{Q}$ ), but the dispersion obtained from $J_{l}(Q, \omega)$ will always have higher energies, because the excitation peaks (or shoulders) are broad and are shifted to higher energies by multiplication with $\omega^{2}$. It should be noted that the dispersion obtained by fitting the frequently used model of the damped harmonic oscillator (DHO) to the inelastic part of $S(Q, \omega)$, corresponds to the dispersion obtained from $J_{1}(Q, \omega)$. Likewise, the dispersion obtained from fitting Bloch's spectral function, $F(Q, \omega)$,

$$
F(Q, \omega)=\left\langle\sum_{j}\left(\overrightarrow{\hat{Q}} \cdot \vec{e}_{j}(\vec{Q})\right)^{2} \delta\left(\omega-\omega_{j}(\vec{Q})\right\rangle\right.
$$

(where $\vec{e}$ is the polarization vector of the excitation, $\overrightarrow{\hat{Q}}$ is the unit vector in direction of $\vec{Q}, \omega_{j}(Q)$ is the dispersion branch $\mathrm{j}$ and $\langle\ldots\rangle$ indicating the ensemble average) corresponds to the dispersion determined from $J_{1}(Q, \omega)$ (see below). As equation 24 demonstrates, $F(Q, \omega)$ is the obvious choice in order to define from its maxima the dispersions of collective excitations in topologically disordered solids [15]. In figure 3 the spectra of the (symmetrized) density- and of the current correlation are compared with the spectral function for identical data.

In computer simulation it is not difficult to determine $F(Q, \omega)$ and even $f_{i j}(Q, \omega)$, the partial spectral functions for a multi-component system and in fact it is highly desirable while investigating the collective excitations in disordered solids that simulation and experiment should meet at the spectral function. However, the experimental determination of $F(Q, \omega)$ (related to the one -excitation part of the recorded intensity only) from even a well corrected intensity involves different laborious subsequent corrections (even in a most simple approach discussed below), which all can only be performed within approximations:

$$
\begin{aligned}
I\left(\overrightarrow{k_{0}}, \vec{k}\right) & \sim\left[S_{\exp }(Q, \omega)\right] * R(Q, \omega), \\
S_{\exp }(Q, \omega) & =S(Q, \omega)+\left(S_{\mathrm{s}}(Q, \omega)\right), \\
S(Q, \omega) & =\frac{4 \pi}{\sigma^{\mathrm{sc}}} \sum b_{i} b j \sqrt{c_{i} c_{j}} S_{i j}(Q, \omega), \\
S(Q, \omega) & =S_{1 \text { phon }}(Q, \omega)+S_{\text {mult.phon }}(Q, \omega),
\end{aligned}
$$




$$
\begin{aligned}
S_{1 \text { phon }}(Q, \omega) & =\frac{1}{\hbar \omega\left(1-\mathrm{e}^{-\beta}\right)} \frac{\hbar^{2} Q^{2}}{\bar{M}} \mathrm{e}^{-2 \bar{W}} F(Q, \omega), \\
F(Q, \omega) & =\sum_{i j} \mathrm{e}^{W_{i}+W_{j}} \sqrt{\frac{c_{i} c_{j}}{M_{i} M_{j}}} f_{i j}(Q, \omega) / \sum_{i j} \mathrm{e}^{W_{i}+W_{j}} \sqrt{\frac{c_{i} c_{j}}{M_{i} M_{j}}},
\end{aligned}
$$

where $\bar{W}(Q)$ are mean Debye-Waller factors. Of these corrections, the de-convolution of the resolution function, $R(Q, \omega)$, is usually not performed. Instead the model used to fit the final data in order to obtain the energy and width of the collective excitations, is convoluted with $R(Q, \omega)$. To subtract the self-part of the dynamic structure factor (incoherent scattering) one has to use a model, e.g. the multi-phonon expansion of $S_{\mathrm{s}}(Q, \omega)[16]$, based on the known vibrational density-of-states, $g(\omega)$, and calculate $S_{\mathrm{s}}(Q, \omega)$ in the harmonic approximation

$$
\begin{aligned}
S_{\mathrm{s}}(Q, \omega) & =A \delta(\omega)+\mathrm{e}^{-2 W(Q)} \sum_{n=1}^{\infty} \frac{f_{n}(\omega)}{n !}\left(\frac{\hbar Q^{2}}{2 M}\right)^{n}, \\
f_{n}(\omega) & =\int_{-\infty}^{\infty} f_{n-1}\left(\omega^{\prime}\right) f_{1}\left(\omega-\omega^{\prime}\right) \mathrm{d} \omega^{\prime}, \\
f_{1}(\omega) & =\frac{g(\omega)}{\hbar \omega\left(1-\mathrm{e}^{-\beta}\right)} .
\end{aligned}
$$

Here $A \delta(\omega)$ represents the elastic scattering. The multi-phonon part of these equations $(n>2)$ can finally be used in order to calculate (in the incoherent and harmonic approximation) and subtract the multi-excitation part, $S_{\text {mult.phon }}(Q, \omega)$ of the coherent scattering. The remaining oneexcitation-part, $S_{1 \text { phon }}(Q, \omega)$ is then directly proportional to $F(Q, \omega)$, which still is a weighted sum over the partial spectral functions, $f_{i j}(Q, \omega)$, in case of a multi-component system.

Inverting the relation between $S_{1 \text { phon }}(Q, \omega)$ and $F(Q, \omega)$ (see equation 26) and taking the BoseEinstein occupation factor in its high temperature approximation leads to a relation completely the same as that of $J_{1}(Q, \omega)$ and $S(Q, \omega)$, except that the equation still contains the Debye-Waller factors and the mass of the scattering unit and the one-excitation part of $S(Q, \omega)$ in place of $S(Q, \omega)[17]$

$$
\begin{aligned}
& F(Q, \omega)=\frac{2 M}{\hbar^{2} Q^{2}} \hbar \omega\left(1-\mathrm{e}^{-\beta}\right) \mathrm{e}^{2 W(Q)} S^{1 \mathrm{phon}}(Q, \omega), \\
& F(Q, \omega) \approx\left(\frac{2 M}{\hbar Q^{2}} \mathrm{e}^{2 W(Q)}\right)\left(\frac{\omega}{Q}\right)^{2} S^{1 \mathrm{phon}}(Q, \omega) .
\end{aligned}
$$

\section{Instruments}

Dispersions of collective excitations in fluids and later topologically disordered solids have been determined using INS by triple-axis and by time-of-flight spectrometry since the second half of the 1960s. Both methods have remained the main sources of information on the collective dynamics in disordered systems using INS (see e.g. [17]). Even though occasionally other types of spectrometers have been used such as a Neutron Spin-Echo (NSE) spectrometer, we will focus here on the two most frequently used spectroscopic methods.

In general, the attempts to measure the dispersion of collective modes at small wave-vector transfers Q turn out to be extremely difficult. To measure the linear part of a dispersion, the velocity, $v_{0}$ of the incident neutrons has to exceed the sound velocity, $v_{\mathrm{s}}$ (optimally by a factor of $\sqrt{2}[18])$ in order to 'cover' the dispersion in the $Q-\omega$-space of the experiment. This high incident energy considerably decreases the good energy resolution available in INS experiments at smaller $E_{0}$. The smallest $\mathrm{Q}$ at high incident $k_{0}=m v_{0} / \hbar$ are only reached at the smallest scattering angles, as shown in equation 3 . Under these conditions one has to measure an exceptionally small signal (because the static structure factor in the low $\mathrm{Q}$ range is of the order of $1 \%$ of its maximal value) aside from the high background next to the incident beam (due to the small scattering angles) 


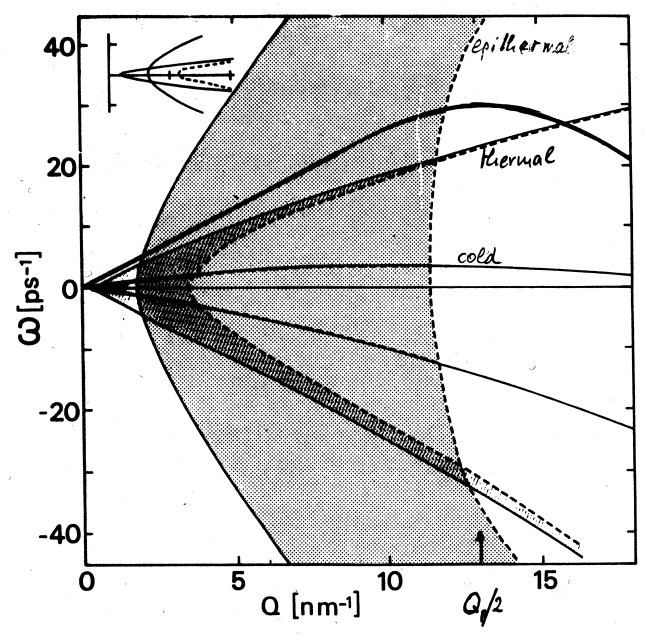

Figure 4. $Q-\omega$-space of Neutron Brillouin Scattering experiments with incident energies of 2.5 (dark grey), 25 (light grey) and $250 \mathrm{meV}$ for scattering angles between 1 and 6 degrees. The dispersion of collective excitations in a solid, e.g., with a sound velocity of $4000 \mathrm{~m} / \mathrm{s}$ can only be measured with the highest incident energies. Insert: enlarged small $Q$ section.

- and this is at a considerably larger distance from the sample (to separate the scattered from the incident beam) than one would do in normal INS experiments. At the same time, the usual multiple scattering intensity, which normally is relative structureless in this Q-range, makes up about $40 \%$ of the recorded intensity leading to large multiple scattering corrections. To meet all these contradicting conditions, the spectrometers and the sample containers (insertions of absorbing grids to reduce the multiple scattering) have to get a special set-up for this kind of experiments.

In triple-axis-spectrometer (TAS) experiments, $\overrightarrow{k_{0}}$ and $\vec{k}$ are determined via Bragg reflections from two single crystals, the monochromator, which selects the desired $\vec{k}_{0}$ from the incident 'white' neutron beam in the direction of the sample outside the monochromator shielding, and the analyzer, which in the same way determines the $\vec{k}$ of the scattered beams (see figure 5 ). The transmitted bandwidths $\Delta k_{0}$ and $\Delta k$, and hence the $Q-\omega$ resolution ellipsoid of the spectrometer, are determined essentially by the mosaic spread of the single crystals and the collimation chosen along the flight path. For NBS experiments, the paths of the neutrons next to the sample are evacuated or flushed with $\mathrm{He}$, which scatters and absorbs neutrons less than air. The advantage of the TAS is its large flexibility

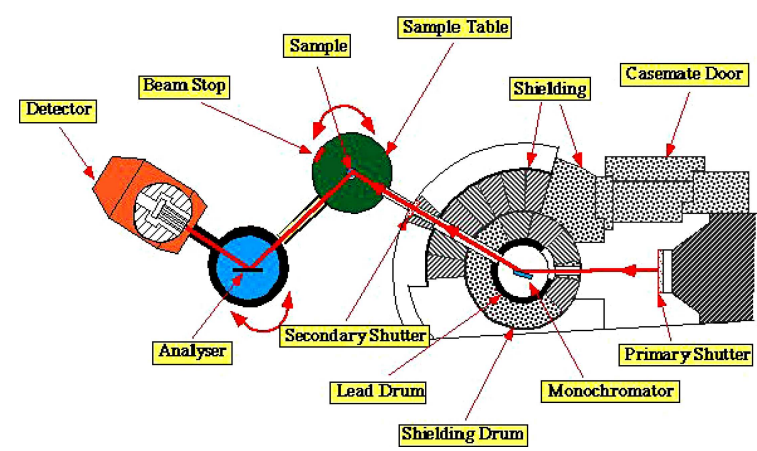

Figure 5. The principle of the IN1 triple.axis spectrometer at the High Flux reactor of the Institut Laue-Langevin in Grenoble. In an NBS-experiment, a large cylindrical vacuum container would be mounted on the sample table and the analyzer would be behind the sample, very few degrees deviating from the direction of the incident neutrons.

(within the accessible kinematic range (see figure 4)) and relative low background due to the numerous collimators and good detector shielding. The flexibility permits to take data (within the kinematically accessible region) at fixed energy- or fixed wave-vector transfer, which frequently makes it possible to immediately interpret the data. However, each point of these cuts at $\omega$ or $Q=$ constant has to be measured with a different configuration of the spectrometer, thus making both quantitative corrections and absolute measurements more difficult. In addition, while the high flexibility is a great advantage, if only certain regions of 
$S(Q, \omega)$ have to be measured, as is the case in phonon dispersion measurements based on a good model for the dynamics of the system under investigation, for topologically disordered systems, due to the weak and broad excitations maxima, $S(Q=$ const, $\omega)$ has to be scanned in a complete energy region, requesting very long measuring times for these NBS experiments on TAS, especially because most of these instruments today still work with a small detector surface [19].

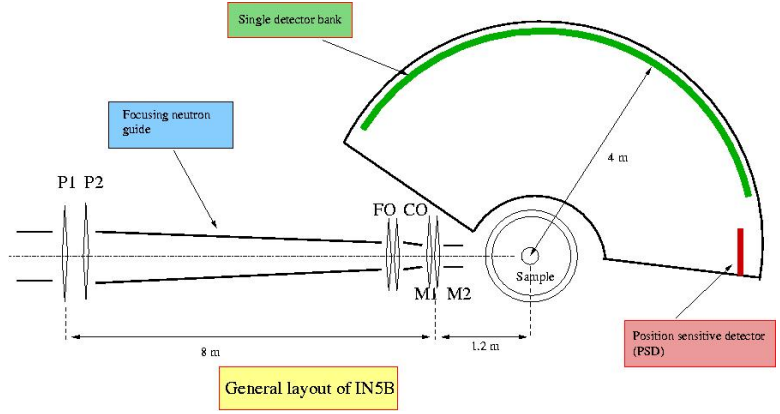

Figure 6. Principle of the new IN5B time-of-flight spectrometer at the High Flux reactor of the Institut Laue-Langevin in Grenoble. For NBS-experiments normally only the two dimensional position sensitive (pixel) detector will be used, permitting to collect the complete intensity of a small angle scattering cone. The flight path of nearly $4 \mathrm{~m}$ is evacuated or flushed with He.

This is different in time-of-flight (TOF) spectroscopy, because there all accessible points of $S(Q, \omega)$ are measured at the same time and under the same experimental conditions without any change of the initially chosen parameters of the experiment. This makes quantitative corrections and the measurement of $S(Q, \omega)$ on absolute scale easier. In TOF spectrometry, $\overrightarrow{k_{0}}$ is determined either by several choppers (see figure 6), which finally let only a small energy band $\Delta E_{0}$ pass onto the sample or (in hybrid TOF spectrometers) by one (or several) monochromator crystal such as in a TAS and one or several choppers, producing neutron pulses with the desired mean $\overrightarrow{k_{0}}$ incident on the sample. The pulses are required, because the modulus of $\vec{k}$ is determined from the timeof-flight of the scattered neutrons between the sample and the large detector area while its direction is determined from the (in NBS experiments: small) scattering angle. The advantage of simultaneous measurements of the scattered intensity at all accessible $Q$ and $\omega$ values is counterbalanced by the fact that the pulsing reduces, in the mean, the incident intensity by a factor of 100 to 200 in comparison with the continuous flux at a TAS. In spite of this, the complete coverage of the interesting part of $S(Q, \omega)$, which permits to 'follow' the dispersion even if the maxima are getting broad at lager $\mathrm{Q}$, the determination of absolute values for the comparison with theories and especially the collection of the complete intensity of the scattering cone at smaller scattering angles on the large position sensitive detector in modern TOF and especially NBS spectrometers, makes the TOF method very attractive for the investigations of collective excitations in disordered matter.

The energy resolution of the elastically scatter neutrons $\mathrm{d} E_{0}$ is of the order of 2 to $3 \%$ on TOF [20,21] and TAS [22,23], - except in the very rare cases e.g. when a TAS is used with a NSEequipment, achieving then an energy resolution down to $1 \mu \mathrm{eV}[24,25]$. The energy resolution in the neutron energy gain spectra $(\omega<0)$ normally deteriorates with the increasing energy transfer, which has to be taken into account when fitting the peaks caused by collective excitations. For the neutron energy loss spectra, the resolution varies little in TOF spectrometry, improving slowly toward larger energy transfers, being a further reason - besides the intensity argument discussed above - for the preference for these spectra.

IXS experiments, due to the very high incident energy of up to $21 \mathrm{keV}$, do not suffer from the kinematic restrictions discussed above for INS. In addition, except for dispersions situated in the range of a few meV such as for compressed gases [13], the resolution down to $1.6 \mathrm{meV}$ (using the $\mathrm{Si}(11,11,11)$ reflection) is very good compared to (non-NSE) INS experiments with high incident energies, though the shape of the resolution function of the IXS backscattering spectrometer crystals (Lorentzian) is not very favorable. Due to the extremely long flight-path of the incident photons, forced by the backscattering geometry, the experiments need about the same time with IXS and INS. Even though it is more difficult to determine $S(Q, \omega)$ on absolute scale, very good results have been obtained for disordered systems using IXS $[3,4,26]$. In spite of all theses advantages, IXS works well only on well selected samples, and therefore one has to expect that 
INS and IXS will remain complimentary methods in the investigation of collective excitations in disordered systems for some time in future.

\title{
References
}

1. Vacher R., Courtens E. Brillouin Scattering. - In: International Tables of Crystallography. Vol. D 'Physical Properties of Crystals'. Ed. Authier A., Kluver, Dordrecht 2003, p. 329 - 335

2. Rat E., Foret M., Massiera G., Vialla R., Arai M., Vacher R., Courtens E., Phys. Rev. B, 2005, 72, 214204.

3. Ruocco G., Sette F., J. Phys.: Condens. Matter, 2001, 13, 9141

4. Scopigno T., Ruocco G., Rev. Mod. Phys., 2005, 77, 881

5. Suck J.-B., Experimental Investigations of Collective Excitations in Disordered Matter. -In: Collective Dynamics of Nonlinear and Disordered Systems Ed. Radons G., Häussler P., Just W., Springer Berlin, Heidelberg 2005 p. $147-170$

6. S.W. Lovesey: Theory of Neutron Scattering from Condensed Matter, (Clarendon Press, Oxford 1984)

7. Copley J.R.D., Verkerk P., van Well A.A., Fredrikze H., Comp.Phys.Commun., 1986, 40, 337

8. J.-P. Boon, S. Yip: Molecular Hydrodynamics, (McGraw-Hill New York 1980)

9. Suck J.-B., Report KFK $1975 \mathbf{2 2 3 1}$

10. Rahman A., Phys.Rev.Lett. 1974 32, 52

11. van Well A.A., Verkerk P., de Graaff L.A., Suck J.-B., Copley J.R.D., Phys.Rev. A, 1985, 31, 3391

12. Pratesi G., Suck J.-B., Egelstaff P. A. J. Non-Cryst. Solids 1999 250-252, 91

13. Suck J.-B., J. Phys.: Condens. Matter, 1991, 3, F73

14. Chushak Ya., Bryk T., Baumketner A., Kahl G., Hafner J., Phys.Chem.Liq. 1996 32, 87

15. Hafner J.: From Hamiltonian to Phase Diagrams, (Springer, Berlin, Heidelberg, New York, London,Paris, Tokyo 1987)

16. Sjölander A., Ark.Fys. 1958 14, 315

17. Suck J.-B., Int. J. Mod. Phys. B, 1993, 7, 3003

18. Robinson R.A., Physica B 1989156 - 157, 557

19. Kempa M., Janousofa B., Saroun P., Flores P., Boehm M., Demmel F., Kulda J., Physica B 2006 385-386, 1080

20. Copley J.R. D., Neut.Instr.Meth.Phys.Res. A 1990 291, 519

21. Jahn S., Suck J.-B., Appl. Phys. A 2002 74, S1465

22. Popovici M., Stoica A.D., Ionita I., J.Appl. Cryst. 1987 20, 90

23. Saroun J., Kulda J., Neutr.News 2002 13, 1573

24. Zeyen C.M.E.., J.Phys.Chem.Solids 1999 60, 1573

25. Kulda J., Farhi E., Zeyen C.M.E., , Physica B 2002 316-317, 383

26. Scopigno T., Suck J.-B., Angelini R., Albergamo F., Ruocco G.,, Phys.Rev.Lett. 2006 96, 135501

\section{Непружнє розсіяння нейтронів в застосуванні до досліджень колективних збуджень у топологічно невпорядкованих системах}

\author{
Й.-Б.Сук \\ Інститут фізики, Технологічний університет Хемнітца, Хемнітц, Німеччина \\ Отримано 22 листопада 2007 р., в остаточному вигляді - 17 грудня 2007 р. \\ Методику непружнього розсіяння нейтронів представлено тут як одну з найважливіших експери- \\ ментальних методик для дослідження колективних збуджень у плинах (рідинах та стиснутих газах) \\ та аморфних твердих тілах. Коротко обговорюються відповідні кореляційні функції, спектри яких \\ визначаються в експериментах по непружньому розсіянню нейтронів, дисперсійні співвідношення \\ для колективних збуджень та як вони можуть бути отримані з вимірюваних спектрів, а також - дві $з$ \\ найбільш часто використовуваних методик.
}

Ключові слова: непружнє розсіяння нейтронів, кореляційні функції, дисперсійні співвідношення, колективні збудження, топологічно невпорядковані системи

PACS: $61.12 . E x, 63.20 . D j, 63.50 .+x$ 